\title{
Stehen wir heute mitten in einer Leserevolution und Liebesrevolution?
}

Wir leben zu Beginn der zwanziger Jahre des 21. Jahrhunderts nicht nur im Westen, sondern in vielen unserer Gesellschaften auf diesem Planeten in einer Epoche rasch fortschreitender Digitalisierung, welche unsere Gewohnheiten und Standards gerade auch des Lesens und der Lektüre grundlegend verändert hat und noch weiter verändert. Die Tatsache, dass wir einen immer höheren Prozentsatz all jener Texte, die wir tagtäglich durcharbeiten, nicht mehr als Druckerzeugnis lesen, sondern immer stärker in einer wie auch immer digitalisierten Form wahrnehmen, verändert unsere Lesegewohnheiten fundamentaler, als wir dies bisweilen wahrhaben wollen.

Es kann daher aus meiner Sicht kein Zweifel mehr daran bestehen, dass wir uns mitten in einer Leserevolution befinden. Diese Leserevolution betrifft das Transportmedium, auf dem wir unsere Texte wahrnehmen, aber natürlich auch die Orte, wo wir lesen, und die Kontexte, in welchen wir Lektüren angehen und unternehmen. Selbstverständlich bleiben auch die Größendimensionen jener Texte, die wir alltäglich lesen, davon nicht unberührt. Dies sind Erfahrungen, wie man sie sehr leicht in jedem Literaturseminar machen kann; längst sind die Zeiten vorbei, zu denen man ganz selbstverständlich dreitausend gelesene Seiten zu Beginn einer Veranstaltung voraussetzen konnte. Verstehen Sie mich nicht falsch! Dies ist keine der zahllosen Klagen über das Lesepensum von Studierenden heutzutage. Die Dinge sind, beschleunigt durch den Bologna-Prozess, ${ }^{1}$ nicht schlechter, sondern anders geworden; genau darauf möchte ich hinaus!

Dies sind Einschätzungen, welche sich auch im unmittelbaren Umfeld wahrnehmen lassen: Die Fähigkeit, lange literarische wie literaturwissenschaftliche Texte zu lesen und kognitiv zu erfassen, ist bei einer keineswegs geringen Zahl an Studierenden gesunken. Denn die Tatsache, dass in immer höherem Maße kurze und kürzeste Texte auf Bildschirmen am Computer zuhause, auf dem iPad an der Universität oder in öffentlichen Transportmitteln auf dem Smartphone gelesen werden, lässt die Fähigkeit, sich über einen längeren Zeitraum zu konzentrieren, ganz ohne Zweifel schwinden. Es scheint mir wichtig, diesen Prozess nicht nostalgisch, zornig oder gar strafend zu begleiten, sondern die veränderten Fähigkeiten im Umgang mit Texten nicht zuletzt auch an der Universität dynamisch

1 Vgl. Ette, Ottmar: Exzellenz(en), velociferische. Zum Bestiarium blendender Bologna-Eliten. In: Horst, Johanna-Charlotte / Kaulbarsch, Vera / Kreuzmair, Elias / Kuhn, Léa / Severin, Tillmann / Tkachenko, Kyrylo (Hg.): Unbedingte Universitäten. Bologna-Bestiarium. Zürich - Berlin: diaphanes 2013, S. 105-110.

Ә Open Access. (C) 2020 Ottmar Ette, publiziert von De Gruyter. (c) BY-NC-ND Dieses Werk ist lizenziert unter der Creative Commons Attribution-NonCommercial-NoDerivatives 4.0 International Lizenz. https://doi.org/10.1515/9783110665093-026 
$\mathrm{zu}$ betrachten und für die Lehre entsprechend $\mathrm{zu}$ nutzen. Denn ja, wir stecken mitten in einer Leserevolution, deren Ziele, Enden und Folgen noch lange nicht absehbar sind! Wird der Mensch am ,Ausgang' dieses Prozesses noch in der Lage sein, sein kulturelles Erbe noch immer speichern und selbst nach Jahrhunderten inhaltlich unverändert abrufen zu können?

Aus der Logik unserer Vorlesung stellt sich auch eine nicht weniger dringliche Frage: Stecken wir zudem in einer Liebesrevolution? Um diese etwas beunruhigende Frage ganz direkt und vorab zu beantworten: Ich meine ja! Denn die gesellschaftlichen, technischen und kulturellen Kontexte unserer Liebesbeziehungen haben sich grundlegend gewandelt und verändern sich mit beeindruckender Geschwindigkeit weiter. Was bedeutet dies für den discours amoureux, dessen Fragmente in dieser Vorlesung zusammengefügt werden sollten?

Ich meine mit der Aussage, dass wir mitten in einer Liebesrevolution stecken, nicht allein, dass wir in Sachen Liebe etwa für die Aufnahme von partnerschaftlichen Beziehungen mittlerweile digitale Dienste und Apps heranziehen und in Anspruch nehmen können; Liebe wird somit jederzeit und an jedem Ort verfügbar, erreichbar, was auch immer wir unter ,Liebe‘ verstehen mögen. Die Vermittlung von Liebesbegegnungen per Smartphone in unserer unmittelbaren Umgebung ist dabei sicherlich nur ein Element, das gänzlich neue Folgen für unsere Liebesbeziehungen zeitigen wird. Auch die Verfügbarkeit von leicht über das Internet zugänglichen erotischen und pornographischen Bildfolgen konfiguriert nur einen weiteren Punkt innerhalb dieses Horizonts, der gerade auch für Kinder und Jugendliche die Vorstellungswelt gelebter Sexualität und Liebe längst grundlegend transformiert hat. Dabei ist nicht so sehr entscheidend, wie häufig derartige Kanäle benutzt werden, sondern die Tatsache der Zugänglichkeit überhaupt, welche sich auch in einem veränderten Sexualbewusstsein etwa an unseren Schulen widerspiegelt und mit dem Sie als Lehrerinnen oder Lehrer bald konfrontiert sein könnten; denn lange vor der eigenen Pubertät haben unsere Schülerinnen und Schüler eine sehr klare und plastische Anschauung von Liebe als Geschlechtsverkehr und dessen einzuhaltender Grammatik.

Es war ganz gewiss kein Zufall, dass Roland Barthes mit seinen Fragments d'un discours amoureux eine Analyse des Liebesdiskurses unternahm, die ohne jede Konkretisierung von Geschlechteridentitäten auskam; dabei war es im Grunde gleichgültig, ob er auf Texte und Vorstellungen der Antike, des Hochmittelalters oder der Jetztzeit zurückgriff. Die Tatsache einer Nicht-Markierung der Geschlechteridentität der Figur seines Liebenden war der entscheidende Schritt hin zu einem neuen Verständnis von Liebesbeziehungen, auch wenn es dem französischen Zeichentheoretiker und Semiologen selbst nicht bewusst sein mochte. 
Gewiss haben wir im Verlauf unserer Vorlesung ,traditionelle` Liebesverhältnisse historisch bedingt stärker in den Vordergrund gerückt; doch ich hoffe, dass Sie durch unseren theoretischen Vorspann einen anderen, offeneren Zugang auch zu diesen tradierten Liebesbeziehungen erhalten haben!

Im Zusammenhang der Fragmente eines Diskurses der Liebe war das autobiographische Faktum keineswegs ausschlaggebend, dass Roland Barthes selbst Homosexueller war; die Offenheit seines geschlechterspezifischen Liebesdiskurses quer durch alle literarhistorischen und kulturgeschichtlichen Epochen hindurch ermöglichte ein freieres, ja ein freies Spiel dieser diskontinuierlichen Inseln und Archipele von Liebesdiskursen, die gerade für unsere Gegenwart in den westlichen Gesellschaften oder, um Denis de Rougemont zu zitieren, für unser Verhältnis zur Liebe im Abendland von entscheidender Bedeutung waren.

Roland Barthes erkannte - bewusst oder unbewusst - mit der ihm zeit seines Lebens eigenen Spürnase für bereits einsetzende und stärker werdende Entwicklungen, dass eindeutige Geschlechteridentitäten zunehmend ins Wanken gerieten; zugleich funktionieren seine Fragmente eines Diskurses der Liebe so, dass die massive Einblendung historischer Liebesdiskurse aus der mittelalterlichen Mystik oder von Goethes Werther in der Gegenwart vergleichzeitigt werden und gleichsam am selben Ort auf Lager liegen und abgerufen werden können. Dies bedeutet nicht zuletzt, dass - ganz im Sinne von Jorge Luis Borges - eine aktuelle Liebespraxis ihre Vorläufer selbst schafft und nicht (nur) von ihren Vorläufern geschaffen wird, so dass aktuelle Liebespraktiken sich bisweilen auch bis ins Gilgamesch-Epos hinein zurückprojizieren vermögen. Literarische Texte sind vieldeutig und viellogisch; sie öffnen sich auf Lektüren der Zukunft und erlauben immer neue, polylogische Bilder der Vergangenheit.

Vor dem längst historisch gewordenen Hintergrund einer sexuellen Revolution in den Ländern des Westens haben sich die Geschlechteridentitäten beizeiten von den biologischen Grundlagen zunehmend fortentwickelt und eröffnen Möglichkeiten, die zuvor als Liebespraktiken nicht nur - wenn überhaupt - versteckt ausgeübt werden mussten, sondern nicht selten auch kriminalisiert wurden und unter Strafe standen. Doch seien wir uns nicht zu sicher! Die historisch erworbenen Spielräume und Freiheiten stehen bei einem politischen Roll-back jederzeit wieder zur Disposition. Die Familienphotos arabischer Familien sprechen da eine eindeutige Sprache: Während die Großmütter Dekolleté trugen, finden wir ihre Töchter und Enkelinnen zunehmend bekleidet und verhüllt auf jüngeren Bildern an der Wand vor. Das kleine Beispiel zur Kleiderordnung im Paris des Jahres 2022 von Michel Houellebecq sollte Ihnen zeigen, dass politische Veränderungen selbstverständlich bis in die Kleiderordnung und mehr noch in die Körperpolitiken durchschlagen; denn jederzeit können Leiber wieder zu politischen Körpern, zu Körper-Objekten, gemacht werden. 
Heute kann fraglos ein wesentlich breiteres Spektrum an Liebesbeziehungen, Liebesverbindungen und Liebespraktiken ausgelebt werden, als dies in früheren Zeiten in westlichen Ländern der Fall war; dabei soll nicht übersehen werden, dass es sehr wohl historisch praktizierte Liebesformen gibt, die zum Teil noch immer oder wieder neu in unseren Gesellschaften marginalisiert oder gar kriminalisiert werden. Wir hatten auf unserem Weg durch die unterschiedlichsten Normen und Formen der Liebe gesehen, dass jede Form der Liebe in ihrer Statik wie in ihrer Dynamik eine politische Form und Norm darstellt, und die in einer Gesellschaft ausgeübten Liebespraktiken uns sehr viel über die politische Verfasstheit dieser Gesellschaften auszusagen vermögen. Nicht allein unsere Körper-Leiber stehen im Zeichen von body politics, sondern auch unsere Liebesformen und Liebesnormen insgesamt; wir leben und erleben das Intime in einem hochpolitischen Zusammenhang!

Geschlechteridentitäten können heute in einigen Ländern des Westens, aber keineswegs etwa in allen Staaten auch nur der Europäischen Union relativ frei gewählt werden. Eine mexikanische Kollegin berichtete neulich, dass sie zu Beginn ihrer Seminare an New Yorker Universitäten all ihre Studierenden bittet, mit Hilfe eines kleinen Schildchens kundzutun, wie sie im weiteren Verlauf des Seminars geschlechterspezifisch angesprochen werden wollen. Ich glaube nicht, dass dies schon im Mittleren Westen so einfach wäre! Geschlechteridentitäten sind auf unserem Planeten sehr stark raumbedingt.

Zugleich hat der ostentative Gebrauch von Liebesbeziehungen und Liebesverhältnissen zugenommen. Selbst in rechtsradikalen Parteien haben gleichgeschlechtliche Partnerschaften explizit ihren Platz. Ob dies allerdings bei einer Machtübernahme dieser Parteien dann noch lange Bestand haben würde, sei dahingestellt! Ich für meinen Teil bin davon überzeugt, dass es sich dabei nur um die scheinbar so liberalen Aushängeschildchen handelt, die sofort einkassiert werden, wenn die Macht derartigen Parteien zufällt. Michel Houellebecq hatte hieran in seiner Roman-Vision von der französischen Gesellschaft der Zukunft keinen Zweifel gelassen. Ein „Das haben wir nicht gewollt!“ wird bei einem echten Roll-back hin zu autoritären Parteien und meinetwegen auch nur, illiberalen Demokratien' recht geräuschlos im gesellschaftlichen Nirgendwo verhallen; und kein Schiff irgendeiner Flussschifffahrtsgesellschaft würde dann noch die Gelegenheit bieten, eine mobile Vektopie für derartige Liebespraktiken zu sein.

Selbstverständlich sind auch Tristan und isolde in unseren Gesellschaften quicklebendig; davon konnten wir uns nicht nur in Assia Djebars Roman Les Nuits de Strasbourg mit seinen Anklängen an Gottfried von Straßburg überzeugen! Die tragische Liebe ist Teil (nicht nur) des westlichen Repertoires all jener Liebesfiguren, die wir tagtäglich bilden und nachbilden; zugleich steht $\mathrm{zu}$ vermuten, dass sich mit der ostentativen Zurschaustellung von Liebesbeziehungen 
die Präsenz des amabam amare des Augustinus deutlich erhöht haben dürfte. Ich liebe also nicht so sehr den Partner oder die Partnerin meiner Liebesbeziehung, sondern vielmehr meine eigene Liebe selbst, die ich diesen Liebespartner ${ }^{\star}$ innen entgegenbringe. Denn noch immer spiegelt sich das Ich als kleines Püppchen in der Pupille der Liebenden ...

Wir stecken also, so meine ich, nicht allein in einer Leserevolution, deren Ende offen ist, sondern zugleich mitten in einer Liebesrevolution, deren Ausgang uns nicht weniger Rätsel aufgibt. Damit scheint sich an dieser Stelle ein letztes Mal in unserer Vorlesung ein Kreis zu schließen; Lesen und Liebe, Lektüre und Liebespraktiken sind aufs Engste miteinander verwoben und konstituieren in nicht geringem Maße die Normen und Formen unseres Lebens, unserer Leben. Die weitere Entwicklung dieses Verhältnisses von Lieben und Lesen ist völlig offen; genau dies macht die Lust unseres Lebens, unsere Lust am Leben aus. An meinem Schreibtisch lese ich gerade diesen eingerahmten Satz des Kubaners José Martí: No se canse de amar, no se canse de amar nunca.

Was am Ende unserer derzeitigen Lese- und Liebesrevolutionen stehen wird, wissen wir nicht! Vielleicht aber werden sich noch immer die Liebenden mit jener Lerche aufschwingen, die in der atemberaubenden Schönheit der Troubadourlyrik eines Bernart de Ventadorn in „Can vei la lauzeta mover“ erscheint. Nein, wir wollen am Ende unserer Vorlesung die Studien eines Erich Köhler ${ }^{2}$ ausblenden und die literatursoziologisch relevanten Dimensionen dieses Liedes, das wir uns gesungen vorstellen müssen, einmal überhören! Denn die ästhetische Kraft dieser Liebesverse aus dem 12. Jahrhundert ragt weit in die Zukunft jenes großen Gefühls, das wir Liebe nennen:

\author{
Can vei la lauzeta \\ Can vei la lauzeta mover \\ De joi sas alas contral rai, \\ Que s'oblid' e.s laissa chazer \\ Per la doussor c'al cor li vai, \\ Ai tan grans enveya m'en ve
}

\section{Das Lerchenlied}

\author{
Wenn ich die Lerche sehe, \\ wie sie voller Freude ihre Flügel zu den Strahlen der Sonne trägt, \\ Wie sie sich dann voller Lebenslust \\ Vergißt und fallen läßt, \\ - Weh mir! - wie beneide ich dann
}

2 Vgl. Köhler, Erich: Observations historiques et sociologiques sur la poésie des troubadours. In: Cahiers de Civilisation Médiévale VII (1964), S. 40-51; sowie ders.: Sens et fonction du terme „jeunesse“ dans la poésie des troubadours. In: Mélanges offerts à René Crozet. Poitiers 1966, S. 569-583; sowie schließlich ders.: „Can vei la lauzeta mover“. Überlegungen zum Verhältnis von phonischer Struktur und semantischer Struktur. In: Estudis de llengua i literatura catalanes: Offerts a R. Aranon i Serra en el seu setantè aniversari. Barcelona: Ed. Catalanes 1979, S. 337-349. 
De cui qu'eu veya jauzion,

Meravilhas ai, car desse

Lo cor de dezirer no.m fon.

Ai, las tan cuidava saber

D'amor, e tan petit en sai,

Car eu d'amar no.m posc tener

Celeis don ja pro non aurai.

Tout m'a mo cor, e tout m'a me,

E se mezeis e tot lo mon!

E can se.m tolc, no.m laisset re

Mas dezirer e cor volon.

Anc non agui de me poder

Ni no fui meus de l'or' en sai

Que.m laisset en sos olhs vezer

En un miralh que mout me plai.

Miralhs, pus me mirei en te,

M'an mort li sospir de preon,

C’aissi.m perdei com perdet se

Lo bels Narcisus en la fon.

De las domnas me dezesper!

Ja mais en lor no.m fiarai!

C'aissi com las solh chaptener,

Enaissi las deschaptenrai.

Pois vei c'una pro no m'en te

Vas leis que.m destrui e.m cofon,

Totas las dopt' e las mescre,

Car be sai c'atretals se son.

D'aisso's fa be femna parer

Ma domna, per qu'e.lh o retrai,

Car no vol so c'om deu voler,

E so c'om li deveda, fai.

Chazutz sui en mala merce,

Et ai be faih co.l fols en pon!

E no sai per que m'esdeve,

Mas car trop puyei contra mon.

Merces es perduda, per ver,

Et eu non o saubi anc mai,

Car cilh qui plus en degr'aver,

No.n a ges, et on la querrai

A can mal sembla, qui la ve,
Alle, die ich glücklich sehe,

Und ich wundere mich, daß mein Herz

Nicht sofort vor Sehnsucht zerspringt.

Weh mir! Wie viel glaubte ich von der Liebe zu wissen,

Und wie wenig weiß ich davon!

Denn ich kann nicht anders, als die lieben,

Bei der ich nie etwas erreichen werde.

Mein Herz hat sie mir genommen, und mich selbst,

Und dann sich und damit die ganze Welt.

Und da sie sich mir nahm, ließ sie mir nichts

Als Sehnsucht und ein unstillbares Verlangen.

Nie mehr hatte ich Macht über mich,

Nie mehr gehörte ich mir selbst

Seit der Stunde, da sie mich in ihre schönen Augen sehen ließ:

In einen Spiegel, der mir so sehr gefällt.

Spiegel, seit ich mich in dir spiegelte,

Haben mich die Seufzer aus der Tiefe getötet,

Und ich verlor mich, so wie sich

Der schöne Narziss verlor, in der Quelle.

An den Frauen verzweifle ich;

Nie mehr werde ich ihnen vertrauen.

Stets habe ich mich für sie eingesetzt,

Aber jetzt werde ich sie im Stich lassen.

Weil ich sehe, daß auch nicht eine einzige mir helfen will

Bei derjenigen, die mich vernichtet,

Fürchte ich sie alle und mißtraue ihnen,

Denn ich weiß wohl: Sie sind alle gleich.

Darin läßt sich meine Dame als wahres Weib erkennen

- Und deshalb werfe ich ihr das auch vor -,

Daß sie nicht will, was man wollen soll,

Und das tut, was man ihr verbietet.

Ich bin in Ungnade gefallen,

Und ich habe mich benommen wie der Narr auf der Brücke

Und ich weiß nicht, warum mir das geschieht

- Außer vielleicht, weil ich mich zu hoch verstieg.

Die Gnade ist wirklich verlorengegangen,

Und ich wußte es nicht, bis jetzt.

Denn diejenige, die am meisten Gnade haben sollte,

Hat überhaupt keine - wo soll ich sie jetzt suchen?

Ach! Wie soll man glauben, wenn man sie sieht, 
Qued aquest chaitiu deziron Que ja ses leis non aura be, Laisse morrir, que no l.aon

Pus ab midons no.m pot valer Precs ni merces ni.l dreihz qu'eu ai, $\mathrm{Ni}$ a leis no ven a plazer Qu'eu l'am, ja mais no.lh o dirai. Aissi.m part de leis e.m recre! Mort m'a, e per mort li respon, E vau m'en, pus ilh no.m rete, Chaitius, en issilh, no sai on.

Tristans, ges no.n auretz de me, Qu'eu m'en vau, chaitius, no sai on. De chantar me gic e.m recre, E de joi e d'amor m'escon .
Daß sie diesen unglücklichen Verehrer, Der ohne sie niemals Freude haben wird, Sterben läßt - erbarmungslos?

Da mir bei meiner Dame nichts helfen will, Weder Bitten, noch Mitleid noch mein Recht, Und weil es ihr nicht gefällt, Daß ich sie liebe, werde ich ihr nie mehr davon sprechen. Ich nehme Abschied von der Liebe und ziehe mich zurück, Sie wollte meinen Tod und soll ihn haben.

Und ich gehe, da sie mich nicht zurückhält, Unglücklich ins Exil, weiß nicht, wohin.

Tristan, Ihr werdet nichts mehr von mir bekommen, Denn ich gehe, jämmerlich, weiß nicht wohin. Ich verzichte auf das Singen

Und verberge mich fern der Lebensfreude und der Liebe ${ }^{3}$

3 Quelle der deutschen Übersetzung: (C) Dr. Angelica Rieger. Lehr- und Forschungsgebiet Interkulturelle Studien - Romanistik. RWTH Aachen University, Kármánstraße 17-19, 52062 Aachen. 